\title{
Surface plasmon polaritons on thin-slab metal gratings
}

\author{
I. R. Hooper and J. R. Sambles \\ Thin Film Photonics Group, School of Physics, University of Exeter, Stocker Road, Exeter, EX4 4QL, United Kingdom
}

(Received 14 October 2002; published 9 June 2003)

\begin{abstract}
In a recently published paper [U. Schröter and D. Heitmann, Phys. Rev. B 60, 4992 (1999)] an unexpected result occurred when light was incident upon a periodically corrugated thin metal film when the corrugations on the two interfaces were identical and in phase with each other. It was observed that it was not possible to excite the surface plasmon polariton on the metal surface facing away from the incoming light, and they ascribed this to the lack of a thickness variation within the metal. In this paper a somewhat different interpretation of their results is presented, which shows that the surface plasmon polariton (SSP) is in fact very weakly excited on the transmission side of such structures. It is explained why this coupling is so weak in terms of the cancellation of the evanescent diffracted orders from the two diffractive surfaces and how, by changing the phase between the grating on either surface, this coupling becomes much stronger. An explanation for the observation that SPP excitation on such structures may lead to either transmission maxima or minima is also presented.
\end{abstract}

DOI: 10.1103/PhysRevB.67.235404

PACS number(s): 73.20.Mf, 42.70.Qs, 78.66.Bz, 41.20.Jb

A surface plasmon polariton (SPP) is an electromagnetic excitation at the interface between a material with a negative permittivity (often a metal) and a dielectric. ${ }^{1}$ It consists of a surface charge density oscillation coupled to electromagnetic fields, which are bound to the surface and which decay exponentially into both media. With an appropriate coupling geometry (in-plane momentum matching) SPP's may be excited by incident radiation. This results in resonant coupling and enhanced optical fields, which is one of the reasons why SPP's may be of use in such fields as surface-enhanced Raman spectroscopy or in nonlinear optics. Interest in SPP's has also been heightened in the last few years after it was discovered that they may mediate enhanced transmission of light through arrays of subwavelength apertures in classically opaque metal films. . $^{2,3}$

When light is incident upon a planar metal surface SPP's cannot be excited since the wave vector parallel to the surface of the SPP is greater than that available to the incident radiation. However, by periodically corrugating the metal surface the wave vector of the incident light may effectively gain integer values of the grating vector, and therefore coupling may occur when the wave vector matching condition is satisfied. Since the increase in the wave vector of the incident light occurs due to diffraction, it is the evanescent diffracted orders of the system which excite the SPP's.

If an optically thin metal film bounded by dielectrics is investigated, then it is possible that SPP's may be excited at both metal and dielectric interfaces, and in each case it is the evanescent-diffracted orders (corresponding to diffraction in each bounding dielectric medium) which excite the SPP. It is this case which Schröter and Heitmann recently investigated. ${ }^{4}$ There is also the possibility of exciting coupled SPP's on either side of the metal film, ${ }^{5,6}$ but this will not be discussed here.

The samples Schröter and Heitmann manufactured (and also modeled) consisted of thin metal films approximately 80 $\mathrm{nm}$ thick, which were corrugated on one, or both, surfaces. These films were produced on quartz substrates, with air as the other bounding medium. They then illuminated these samples from the quartz side with a multiwavelength source at angles near normal incidence. They found that when only one surface was corrugated (either surface) SPP's could be excited at both interfaces, whereas if both surfaces were corrugated with identical gratings (in a conformal geometry) only the SPP on the surface irradiated could be excited. If one of the gratings on these doubly corrugated films was phase shifted with respect to the other, then excitation of SPP's on both surfaces was again found to occur.

This modeling has been repeated using a code based upon the same method which they used, but using different grating parameters in order to replicate their results as closely as possible (unlike Schröter and Heitmann a sinusoidal grating profile is used in order to simplify the problem since it removes the possibility of scattering processes from the higher harmonics of the surface profile). This code utilizes an idea first proposed by Chandezon et al., ${ }^{7}$ in which a curvilinear coordinate transformation is used to map the grating onto a flat plane. This approach is chosen as it enables easier matching of the electromagnetic boundary conditions, and therefore the reflection and transmission of complex structures may be calculated. ${ }^{8}$ A polynomial fitted to experimentally determined values ${ }^{9}$ is used throughout this work to describe the frequency-dependent dielectric function of the silver:

$$
\begin{aligned}
\varepsilon_{r}= & -255.3189+1.9863 \times 10^{-13} \omega-6.0794 \times 10^{-29} \omega^{2} \\
& +8.3810 \times 10^{-45} \omega^{3}-4.3004 \times 10^{-61} \omega^{4}, \\
\varepsilon_{i}= & 83.2575-1.3279 \times 10^{-13} \omega+9.0474 \times 10^{-29} \omega^{2}-3.2880 \\
\times & 10^{-44} \omega^{3}+6.6591 \times 10^{-60} \omega^{4}-7.0893 \times 10^{-76} \omega^{5} \\
+ & 3.0913 \times 10^{-92} \omega^{6},
\end{aligned}
$$

where $\omega$ is the angular frequency of the incident light.

The results of these calculations for structures designed to replicate the results of Schröter and Heitmann as closely as possible with normally incident TM-polarized light as a function of frequency are shown in Fig. 1. The grating parameters used in these calculations are the following: the 

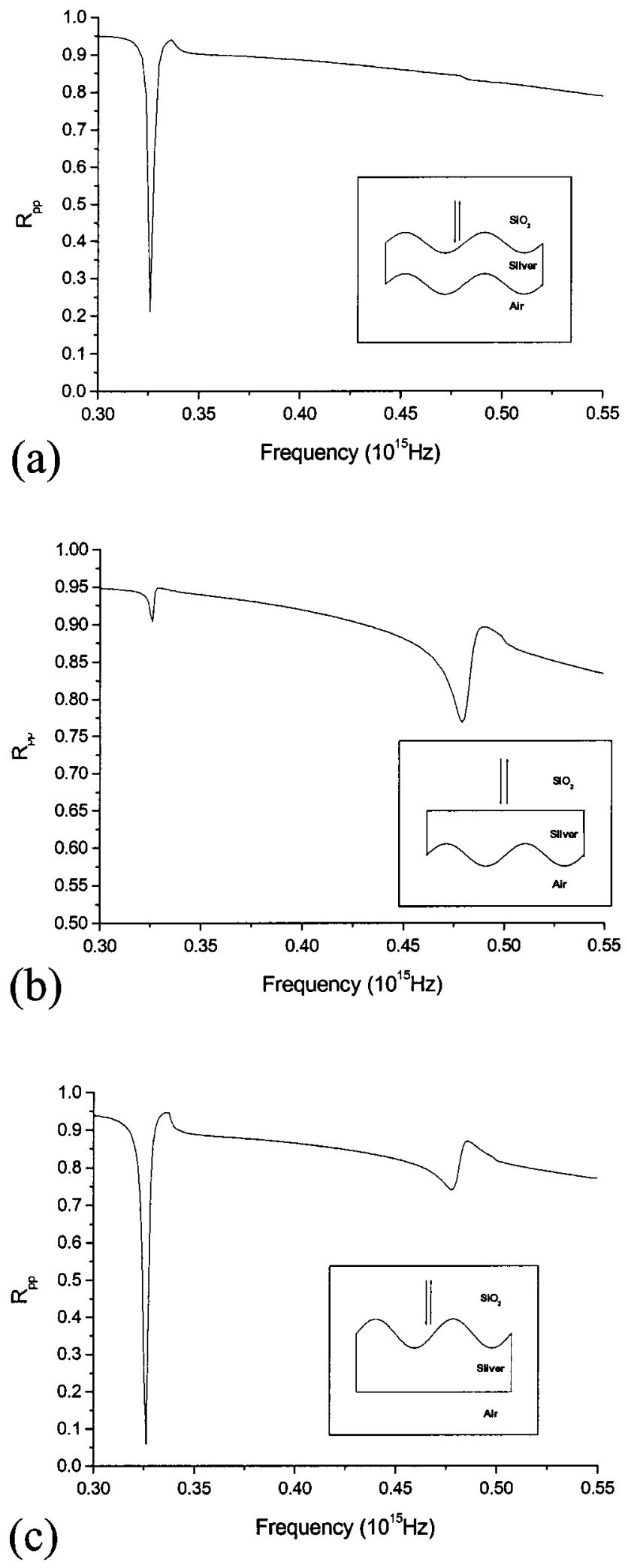

FIG. 1. Reflectivity of $p$-polarized light for normal incidence in the classical mount as a function of frequency for a 40-nm-thick silver film corrugated with a 600 -nm-pitch sinusoidal grating of 10 nm amplitude: (a) corrugated on both sides, (b) corrugated on the bottom surface only, and (c) corrugated on the top surface only. average silver film thickness is $40 \mathrm{~nm}$, the grating pitch is $600 \mathrm{~nm}$, and the sinusoidal corrugations are $10 \mathrm{~nm}$ in amplitude. The results confirm their observations in that the SPP at the air/metal interface (which would be expected to occur at $f \sim 0.475 \times 10^{15} \mathrm{~Hz}$ ) does not appear to be excited on the conformal structure.

The explanation given by Schröter and Heitmann for the lack of coupling to the SPP on the transmission side interface was that a thickness variation in the metal film was necessary for coupling to occur. However, while this is in essence true, they do not explain why it is necessary. Presented in this paper is an extension of their results, which show that the SPP on the nonincident side of the structure is in fact excited, but only very weakly. The reason for this is explained, as is a second phenomenon: the fact that the feature seen in the transmitted zero order can be observed as a transmission maximum, minimum, or as a Fano-shaped resonance.

First, it will be shown that the SPP on the transmission side of the structure is excited even with a conformal geometry. Figure 2(a) shows the zero-order transmission through, and total absorption of, the structure. It is clear from these results that there is a very small transmission maximum at the frequency at which the SPP on the transmission side of the grating would be expected to occur $\left(f \approx 0.48 \times 10^{15} \mathrm{~Hz}\right)$ and that this corresponds to some small resonant absorption of the incident light. Due to the fact that the coupling to this SPP is so weak, it is not surprising that there is no feature in the corresponding reflectivity plot of Fig. 1(a). Since the fields due to the SPP will decay exponentially away from the surface, they will be so weak at the incident side that any scattering from that surface into the specularly reflected order will be too weak to have a noticeable effect on the reflectivity response of the structure. However, to understand why the coupling to the SPP is so weak it is necessary to consider the magnitude and phases of the complex amplitude coefficients of the possible contributions to the transmitted diffracted field which excites it.

Consider a thin metal slab with complex refractive index $n_{2}$, corrugated on both surfaces conformally with a 600-nm pitch grating and bounded on either side with dielectrics of refractive indices $n_{1}$ and $n_{3}\left(n_{1}\right.$ on the incident side and $n_{3}$ on the transmission side). Then, over a certain wavelength range at normal incidence, light will be diffracted at the first interface and produce three transmitted orders: the zeroth and \pm 1 diffracted (which will be evanescently decaying in the $y$ direction). (For this grating pitch and with normally incident light of the frequency range investigated, the other evanescent diffracted orders are extremely small and will be ignored.) There are then nine possible scattered fields transmitted through the slab: the zeroth transmitted order from the first interface may pass through the second interface or be diffracted by it, and the evanescent diffracted orders from the first interface may pass through the second interface or be diffracted by it (Fig. 3). Therefore the resultant transmitted diffracted orders from such a system will be a combination of the diffraction from the two interfaces.

The efficiencies and phases of the +1 transmitted diffracted orders from single interface gratings (both $\mathrm{SiO}_{2} /$ metal and metal/air) can be obtained by using an itera- 

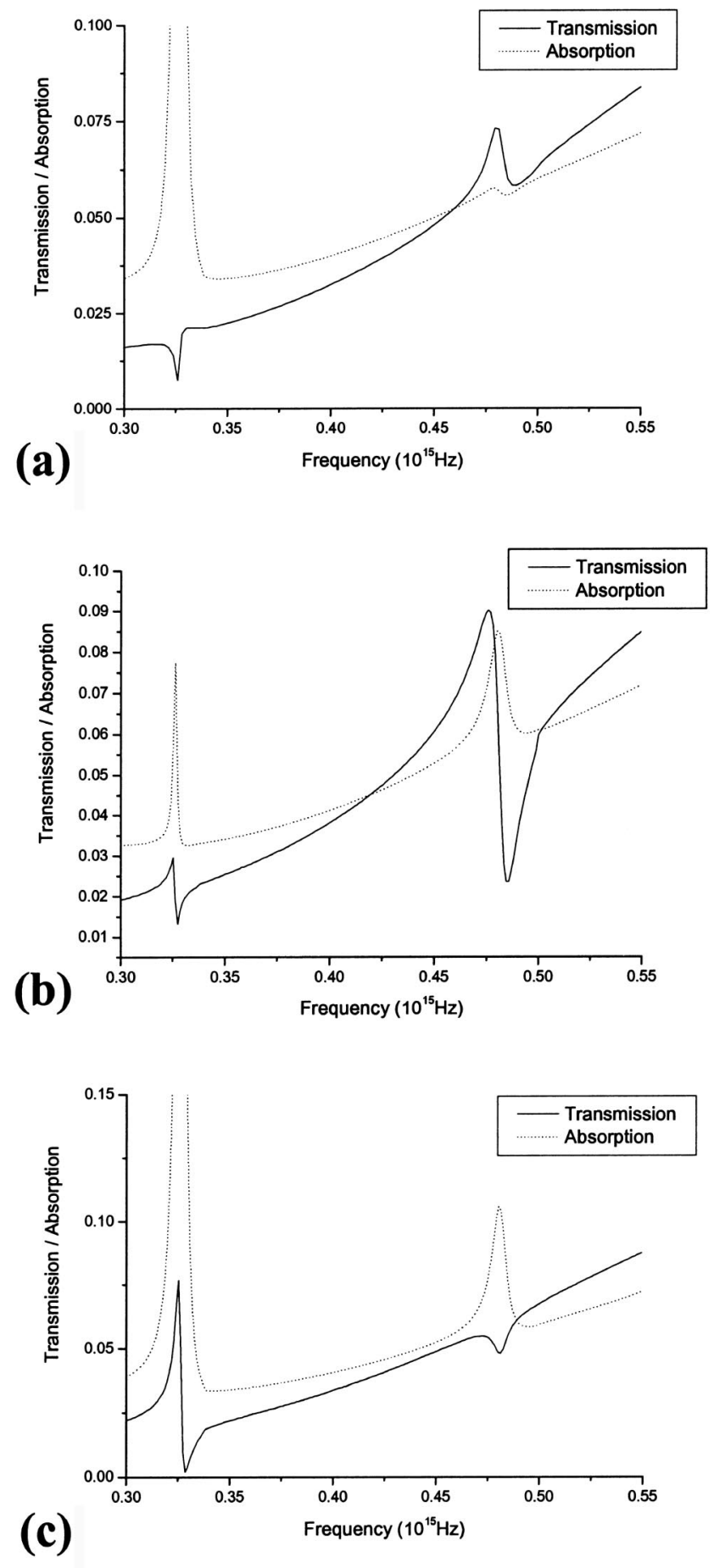

FIG. 2. Transmissivity and absorption of p-polarized light for normal incidence in the classical mount as a function of frequency for the same structures as in Fig. 1.

tive series solution (ISS) method. ${ }^{10-12}$ By combining the results for the evanescent-transmitted diffracted order created at the incident interface, which is not diffracted at the second interface, and the zero-order transmitted field from the incident interface, which is diffracted evanescently at the second interface, the approximate total transmitted +1 diffracted field from a thin metal slab can be obtained (the reasons why

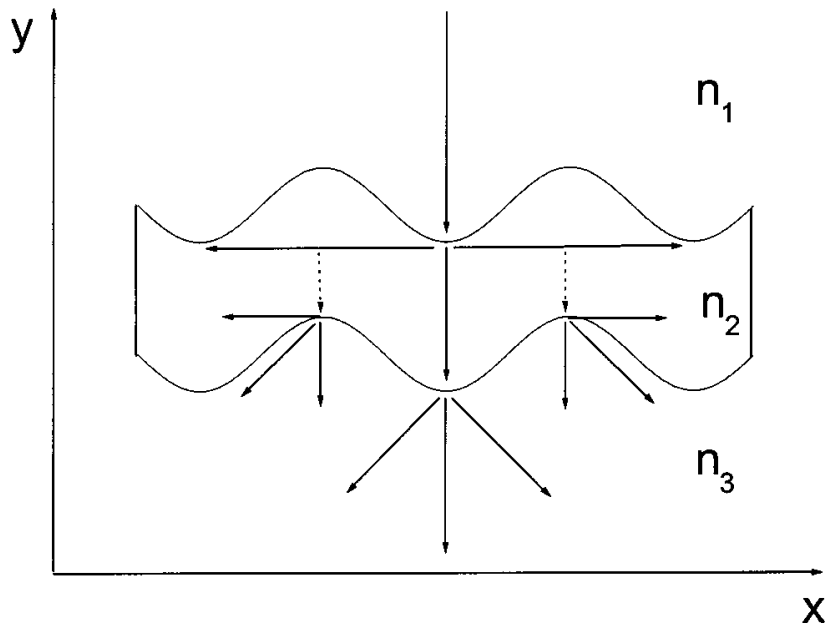

FIG. 3. Schematic showing the origin of the possible transmitted orders due to diffraction from a thin metal slab corrugated on both surfaces. The dotted lines indicate the evanescently decaying fields due to diffraction at the top interface.

this is only approximate will be discussed later). In this method the transmitted or reflected fields are expressed as sums of plane waves (Rayleigh hypothesis) and the scattered fields are related by a scattering potential which is expanded in powers of the surface. The transmitted diffracted order complex amplitude coefficients are then obtained from

$$
e_{t}(m)=\sum_{n=0}^{\infty} \frac{e_{t}^{(n)}(m)}{n !}
$$

where $m$ corresponds to the transmitted order of interest and $n$ is the number of iterations needed to achieve convergence, with the coefficients $e_{t}^{(n)}(m)$ for the transmitted orders (with $p$-polarized light normally incident upon a sinusoidal grating in the classical mount) obtained iteratively from [taken from Eqs. (12)-(15) in Ref. 9],

$$
e_{t}(0)^{(0)}=\frac{2 \sqrt{\varepsilon_{1}}}{\sqrt{\varepsilon_{1}}+\sqrt{\varepsilon_{2}}},
$$

for $n=0$, and

$$
\begin{aligned}
e_{t}(m)^{(n)}= & \frac{k_{0}^{2}\left(\varepsilon_{1}-\varepsilon_{2}\right)}{\gamma_{t}+\gamma_{r}} \sum_{q=1}^{n}\left(\begin{array}{l}
n \\
q
\end{array}\right) i^{q} \frac{h^{q}}{2^{q}} \sum_{k=0}^{q}\left(\begin{array}{l}
q \\
k
\end{array}\right)\left(\gamma_{r}-\gamma_{t}\right)^{q-1} \\
& \times M(m, m+q-2 k) e(m+q-2 k)^{(n-q)}
\end{aligned}
$$

for $n>0$, where

$$
\begin{gathered}
M(m, m+q-2 k)=\frac{\kappa \kappa^{\prime}+\gamma_{r} \gamma_{t}^{\prime}}{\kappa^{2}+\gamma_{r} \gamma_{t}}, \quad \kappa=m k_{g}, \\
\kappa^{\prime}=(m+q-2 k) k_{g}, \\
\gamma_{r}^{2}=\varepsilon_{1} \frac{\omega^{2}}{c^{2}}-\kappa^{2}, \quad \gamma_{t}^{2}=\varepsilon_{2} \frac{\omega^{2}}{c^{2}}-\kappa^{2}, \quad \gamma_{t}^{\prime 2}=\varepsilon_{2} \frac{\omega^{2}}{c^{2}}-\kappa^{\prime 2},
\end{gathered}
$$


and $\varepsilon_{1}$ and $\varepsilon_{2}$ are the complex dielectric functions of the top half space and bottom half space media, respectively, and $k_{g}$ is the grating vector.

To first order the amplitude coefficient of the +1 transmitted diffracted order is given by [from Eqs. (2) and (3)]

$$
e_{t}(1)^{(1)}=\frac{i\left(\varepsilon_{2}-\varepsilon_{1}\right) k_{0}^{3} h \gamma_{r} \sqrt{\varepsilon_{2}}}{2\left(\gamma_{t}+\gamma_{r}\right)\left(k_{g}^{2}+\gamma_{r} \gamma_{t}\right)}\left(\frac{2 \sqrt{\varepsilon_{1}}}{\sqrt{\varepsilon_{1}}+\sqrt{\varepsilon_{2}}}\right),
$$

and this first-order approximation can be used to understand the results of Figs. 1 and 2. In the theoretical modeling shown throughout the rest of this paper the code based on the full ISS method is used; however, this first-order expression will be referred to in order to enable a basic understanding of the physical processes involved.

It must be noted that this method only achieves convergence for gratings with a lower aspect ratio than those studied by Schröter and Heitmann, but does achieve convergence for the profile investigated here. The method is also very limited in that it may only be used to calculate the efficiencies and phases from single interface structures, unlike the Chandezon method. Therefore, combining the diffracted order efficiencies in the way described above will not give the total transmitted diffracted field for these thin-slab structures since it does not account for the multiple reflection and scattering processes within the thin film. However, since all orders within the metal film are exponentially decaying and, therefore, higher-order contributions should be small, this method can be used as a close approximation since its simple analytical form may enable a better physical understanding of the processes involved than the Chandezon method. It also allows the various scattering processes to be calculated individually, which may facilitate a better understanding of the phenomena investigated.

Figures 4(a) and 4(b) show the magnitudes and phases of the +1 diffracted orders for the two cases described above and also show the magnitude of the total diffracted order for a thin-film structure, obtained by combining these two results (c). These figures show a peak in the magnitude of the complex amplitude coefficient which corresponds to the excitation of the SPP on the lower interface. The phase also undergoes a $180^{\circ}$ phase change through this point due to the fact that the SPP is being resonantly driven by the evanescent diffracted order.

It is clear that the two contributions to the total +1 transmitted diffracted order fields are approximately $180^{\circ}$ out of phase with each other throughout the frequency range investigated. Therefore, when these two contributions are combined they almost cancel each other, leaving only a small transmitted diffracted order field due to the difference in the magnitude of the two contributing diffraction processes and the fact that they are not quite in antiphase. Since the total transmitted diffracted field is very small, only very weak coupling to the SPP occurs, which in turn produces only small features in the zero-order transmission from the structure. As described previously, this produces an even smaller feature in the reflected zeroth order than in the transmitted zeroth order due to the exponential decay of the reradiated light through the silver film.

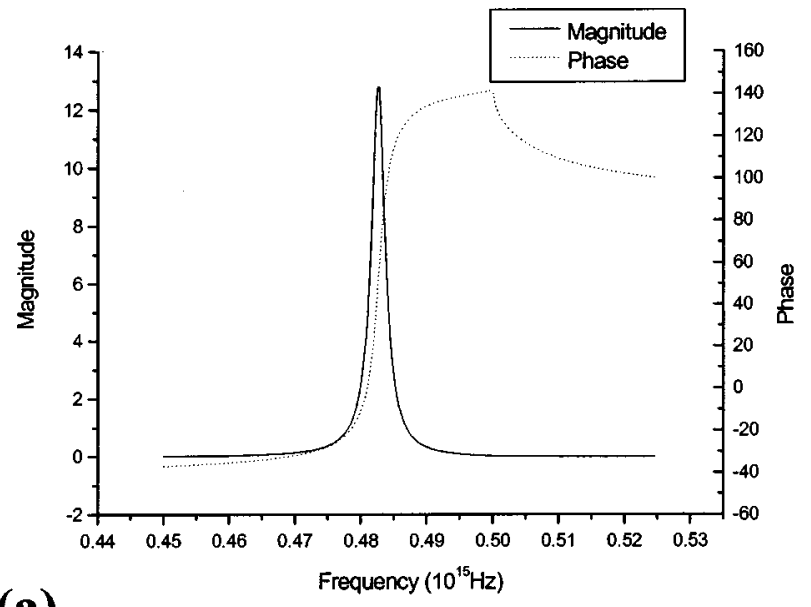

(a)
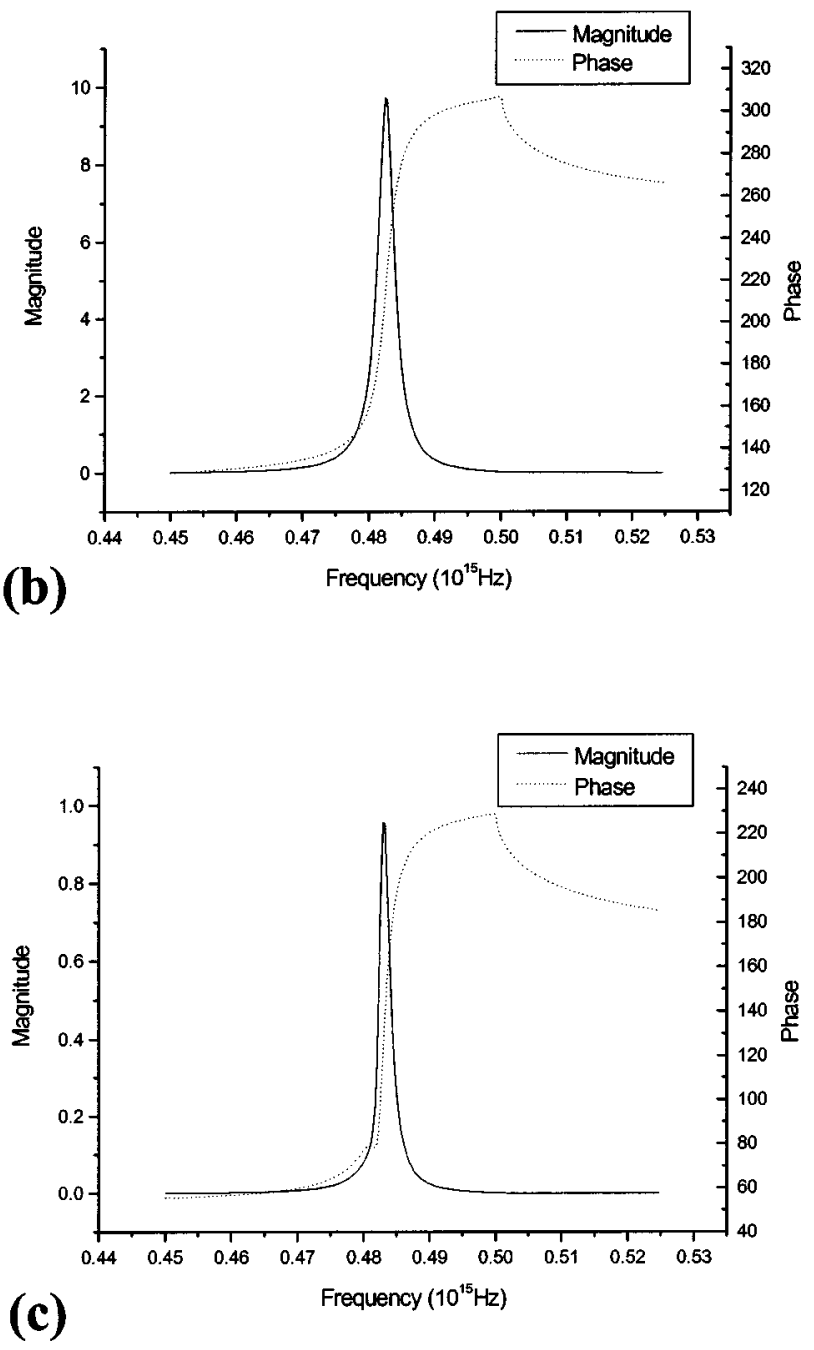

FIG. 4. Magnitude and phase of the first-order transmitted diffracted field around the SPP excitation frequency from a conformally corrugated structure for (a) diffraction from the top surface, (b) diffraction from the bottom surface, and (c) the total first-order transmitted diffracted field. 
This $180^{\circ}$ phase difference can be seen from Eq. (4) where the $\varepsilon_{2}-\varepsilon_{1}$ factor changes sign for the diffraction at the $\mathrm{SiO}_{2} /$ metal boundary compared to that at the metal/air boundary. In fact, it is clear that this must be the case since, as the thickness of a thin film tends towards zero, the resultant diffraction may only be due to any difference between $n_{1}$ and $n_{3}$.

For the other structures investigated by Schröter and Heitmann this cancellation does not occur. It is clear from Fig. 2 that these structures have more strongly coupled SPP's on the transmission side of the structure as evidenced by the increased absorption on resonance. However, the sizes of the features in the transmitted and reflected zeroth orders do not necessarily make this fact obvious. As an example, the flatbottomed structure [Fig. 2(c)] can be compared to the conformal structure [Fig. 2(a)]. It may be expected that the feature in the transmitted zeroth order for the flat bottomed structure should be larger than that of the conformal structure since the SPP is more strongly coupled in this case. This is clearly not true, and in order to understand this it is necessary to consider the propagation of the reradiated light through the silver slab. The SPP on the transmission side can only scatter off of the corrugation on the incident side in the case of the flat-bottomed structure. Therefore, in transmission, there has been an exponential decay of the SPP fields through the silver film before it is scattered by the corrugation on the top surface. This reradiated light is then damped further as it propagates through the silver film into the transmitted zeroth order. These two damping processes mean that the intensity of the reradiated light which produces the feature in the transmitted zeroth order is even smaller than that reradiated from the conformally corrugated structure (even though the energy contained within the SPP for the conformally corrugated structure is much weaker). This then produces a smaller feature in the case of the flat-bottomed structure than for the conformal structure. In reflection the SPP fields and reradiated light from the transmission side only have to propagate through the silver film once for both the conformal and flat-bottomed structures. Therefore, the cancellation of the fields in the conformal case leads to a much smaller feature in reflection [Fig. 1(a)] than in the flatbottomed case [Fig. 1(c)].

When both surfaces are corrugated, but phase shifted with respect to each other, the case is a little more complex. Since the SPP is excited by the evanescent-diffracted order, the fields have a periodicity in the $x$ direction (parallel to the grating vector) only. This periodicity is caused by the diffraction from the grating surface, and the fields produced have their maxima and minima at the maxima and minima of the grating profile. The diffraction occurring at the $\mathrm{SiO}_{2} /$ metal boundary at some instant in time may have field maxima at the maxima of the grating profile from which it is diffracted. If this is the case, then at the same instant in time the diffraction occurring at the metal/air boundary will have field minima at the maxima of the grating from which it is diffracted. This is the $180^{\circ}$ phase difference described earlier. If the phase of the grating on the transmission side is altered with respect to that on the incident side by some phase $\phi$, then, since the field maxima and minima are locked to the
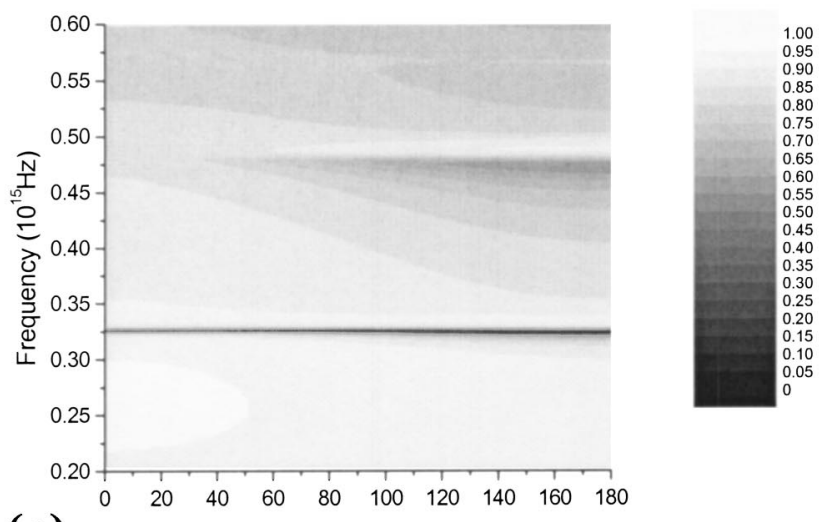

(a) Phase difference between gratings (degrees)
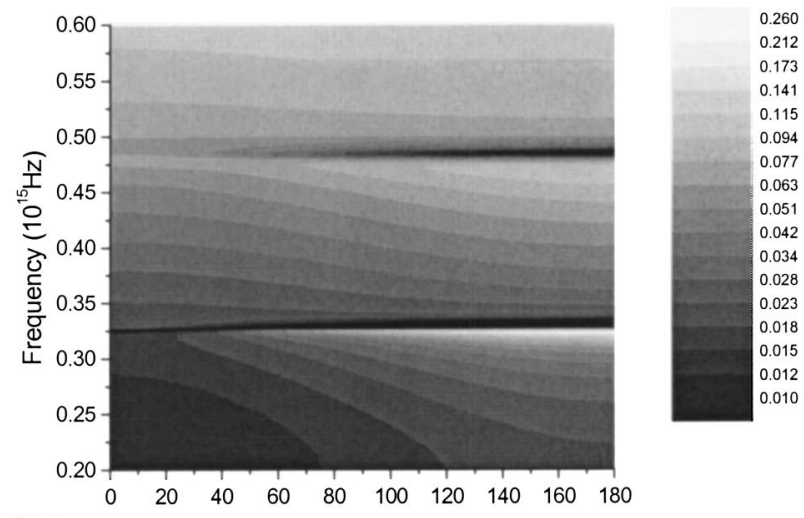

(b) Phase difference between gratings (degrees)

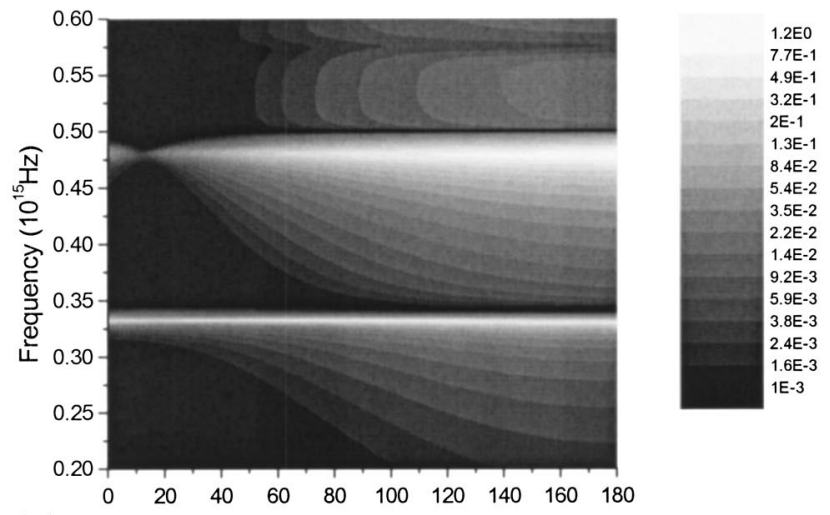

(c) Phase Difference Between Gratings (degrees)

FIG. 5. Results from a dual corrugated silver film as a function of the phase between the corrugation on the two interfaces and frequency: (a) zero-order reflection, (b) zero-order transmission (log scale), and (c) the magnitude of the first diffracted order (log scale).

grating profile from which it is diffracted, the phase difference between the two diffracted orders is now equal to $180^{\circ}-\phi$. Since the phase difference is no longer $180^{\circ}$, the two diffracted orders will not cancel in the same way and stronger coupling to the SPP can occur. This is shown in Fig. 5 , where the zero-order reflectivity and transmissivity are 
shown as a function of frequency and the phase between the two gratings. Also shown is the magnitude of the amplitude coefficient for the +1 diffracted order. It is clear that the coupling to the SPP on the transmission side (at $f \approx 0.48$ $\times 10^{15} \mathrm{~Hz}$ ) generally increases as the phase difference between the two grating surfaces is increased and that this corresponds to an increase in the magnitude of the complex amplitude coefficient. Also to be noted is that the minimum in the magnitude of the amplitude coefficient actually occurs at a phase difference between the two gratings which is not $0^{\circ}$. This is due to additional small phase and amplitude differences between the fields created by the two diffraction processes, which give rise to the total transmitted diffracted field, caused by the thickness of the silver film.

In order to completely understand the results of Schröter and Heitmann the shape of the resonance features observed in the transmitted zero order must also be considered. From Fig. 2 it is clear that transmission maxima or minima may occur on resonance or that a Fano-shaped resonance may occur (one which shows a maximum and minimum on either side of the resonance frequency). In order to understand this it is first necessary to understand how the features due to SPP excitation originate in the reflected and transmitted zero orders.

For light incident upon a semi-infinite metal grating the phase of the $\mathbf{E}$ field of the specularly reflected order is changed by $180^{\circ}$ with respect to the incident light. Also, the evanescent-diffracted orders are $90^{\circ}$ out of phase with the incident light and, due to the fact that the SPP is resonantly driven by the evanescent order (producing another $90^{\circ}$ phase shift), the SPP is in antiphase with the incident light. When light is reradiated from the SPP this process is repeated so that the reradiated light is in antiphase with the specularly reflected order, thereby canceling and producing a reflectivity minimum.

When an optically thin metal film is investigated there is similarly a $180^{\circ}$ phase change from the SPP when it is reradiated into the transmitted zero order. Figure 4(c) shows the resultant diffracted field phase and magnitude for the combined diffraction processes from the top and bottom surfaces of a thin silver film in a conformal geometry, and this shows that the phase on resonance is approximately $140^{\circ}$ with respect to the incident light. Due to the metal film thickness, the phase of the zero order for a planar film with the same average thickness is approximately $-40^{\circ}$, and therefore when the $180^{\circ}$ phase change from the SPP upon reradiation is taken into account these are in phase with each other and result in a transmission maximum. This is as observed in Fig. 2(a).

This same type of analysis can be used to understand the other transmission features observed on the structures investigated in this paper. The transmission minima occur when the reradiated light is in antiphase with the zero-order transmitted light, and the Fano-shaped resonances occur when the reradiated light is $\pm 90^{\circ}$ out of phase with the zeroth-order transmitted light. Of course, this is very much simplified here as in most real cases (for example, when the phase between the two gratings on either surface is not a simple multiple of $\pi / 2$ ) the phase between the reradiated light and the transmitted zero order will not be $0^{\circ}, 90^{\circ}$, or $180^{\circ}$, but rather somewhere between. Also, the shape of the resonance will depend strongly upon the relative intensities between the reradiated light and the transmitted zero order, therefore being strongly dependent upon the film thickness and grating profile. An indication of the change in the features with changing phase of the reradiated light is clearly evident in the results of Fig. 5. As the phase difference between the two corrugations is altered, the phase of the reradiated light with respect to the zeroth orders also alters. Therefore, from the arguments on the resonance feature shapes given above, a change in the shape of the feature would be expected and is clearly evident in the results.

In this paper an explanation of the results of Schröter and Heitmann, ${ }^{4}$ in which they investigated the SPP's of a thin metal film corrugated on both sides, has been presented. They suggested that the SPP bound to the surface facing away from the incident radiation could not be excited at normal incidence since there was no thickness variation through the metal film. A simple approximation considering each of the diffraction processes at the interfaces separately and any phase changes due to propagation through the silver slab has been used to show that the SPP is in fact excited, but that, due to phase cancellation between the diffraction arising from the two interfaces, it is very weakly coupled. Therefore, though their assertion that a thickness modulation is needed is, in essence, true, this paper has given an understanding of the physical processes behind this phenomenon.

If only one surface of the film is corrugated or one of the corrugations is phase shifted with respect to the other, this cancellation is altered enabling stronger coupling to take place. Also, an explanation of the shape of the features in the transmitted zero order due to the excitation of the SPP, where transmission maxima, minima, or Fano-shaped resonances have been found to occur, has also been presented. These different resonance shapes have been shown to be due to the difference in phase between the reradiated light from the SPP and the transmitted zero order.

The authors gratefully acknowledge useful discussions with Professor W. L. Barnes and would also like to thank the EPSRC for financial support and the provision of a CASE award by QinetiQ (Farnborough) for I.R.H. This work was carried out as part of Technology Group 08 of the MoD Corporate Research Fund.

\footnotetext{
${ }^{1}$ H. Raether, Surface Plasmons (Springer-Verlag, Berlin, 1988).

${ }^{2}$ T. W. Ebbesen, H. J. Lezec, H. F. Ghaemi, T. Thio, and P. A. Wolff, Nature (London) 391, 667 (1998).
}

\footnotetext{
${ }^{3}$ H. F. Ghaemi, Tineke Thio, D. E. Grupp, T. W. Ebbesen, and H. J. Lezec, Phys. Rev. B 58, 6779 (1998).

${ }^{4}$ U. Schröter and D. Heitmann, Phys. Rev. B 60, 4992 (1999).
} 
${ }^{5}$ T. Inagaki, M. Motosuga, E. T. Arakawa, and J. P. Goudonnet, Phys. Rev. B 32, 6238 (1985).

${ }^{6}$ S. Dupta Gupta, G. V. Varada, and G. S. Agarwal, Phys. Rev. B 36, 6331 (1987).

${ }^{7}$ J. Chandezon, M. T. Dupuis, G. Cornnet, and D. Maystre, J. Opt. Soc. Am. 72, 839 (1982).
${ }^{8}$ N. P. K. Cotter, T. W. Preist, and J. R. Sambles, J. Opt. Soc. Am. A 12, 1097 (1995).

${ }^{9}$ Handbook of Optical Constants of Solids, edited by E. D. Palik (Academic, Orlando, 1985).

${ }^{10}$ J-J. Greffet and Z. Maassarani, J. Opt. Soc. Am. A 7, 1477 (1990).

${ }^{11}$ J-J. Greffet, Phys. Rev. B 37, 6436 (1988).

${ }^{12}$ A. A. Maradudin, J. Opt. Soc. Am. A 73, 759 (1983). 